\title{
Abstraction and Genericity in Why3
}

\author{
Jean-Christophe Filliâtre and Andrei Paskevich^ \\ Université Paris-Saclay, CNRS, Inria, LRI, 91405, Orsay, France
}

\begin{abstract}
The benefits of modularity in programming - abstraction barriers, which allow hiding implementation details behind an opaque interface, and genericity, which allows specializing a single implementation to a variety of underlying data types - apply just as well to deductive program verification, with the additional advantage of helping the automated proof search procedures by reducing the size and complexity of the premises and by instantiating and reusing once-proved properties in a variety of contexts.

In this paper, we demonstrate the modularity features of WhyML, the language of the program verification tool Why3. Instead of separating abstract interfaces and fully elaborated implementations, WhyML uses a single concept of module, a collection of abstract and concrete declarations, and a basic operation of cloning which instantiates a module with respect to a given partial substitution, while verifying its soundness. This mechanism brings into WhyML both abstraction and genericity, which we illustrate on a small verified Bloom filter implementation, translated into executable idiomatic $\mathrm{C}$ code.
\end{abstract}

\section{Introduction}

When Alice writes code that uses hash tables, she does not need direct access to the actual implementation of that data structure - only to the handful of operations provided by it. Truth be told, she would rather not have that access: less risk to break her data structure by mistake, and she can also swap one implementation for another, provided that the offered operations behave in the same way. What she needs, however, is hash tables for cabbages and hash tables for kings, and hash tables for whatever other data type she has in her code, for which she has written a hash function and an equality test ${ }^{1}$.

If Alice also wants to formally verify her program, then not having access to the implementation may easily become a necessary requirement for her success. The automated provers are more stubborn than smart, and they will happily drown in all the minute properties of the implementation, whereas they could easily succeed in their proof, were they given just the simple specifications of hash table operations. The best way to get an automated proof of anything is to

\footnotetext{
* This research was partly supported by the French National Research Organization (project VOCAL ANR-15-CE25-008) and by the Inria-Mitsubishi Electric bilateral contract "ProofInUse-MERCE".

1 "Cabbage hash can be delicious," said Alice, "but I would never dare to hash a king."
} 
give the prover very little data written in very simple terms (incidentally, this also helps if at some later point you need to slightly change your problem and be able to prove it again). And then, if you do get the proof, just make sure that it somehow still holds if your terms are not as simple and your problem is actually much larger than what you let your prover believe.

What this means for Alice, is that she would prefer to verify her code without knowing anything about how hash tables are implemented, and if she also verified her implementation of hash tables, she would prefer to do it just once, without giving her prover any details about the type of the objects to store, only that there is an equality test and a hash function for them. If her verification framework is done right, this should be enough to guarantee that her final executable - where sophisticated and highly performant hash tables are reused for ships and shoes and sealing-wax sticks — is flawless.

Probably, any approach invented for modularity in programming can be adapted to program verification. The purpose of this work is to show how we do it in WhyML, the language of the program verification tool Why3 [6].

Our framework is inspired by theorem proving just as much as by programming. In classical, non-constructive, logic, the difference between full implementation and partial specification is just how much you say about your type, function, or program. Also, apart from some symbols that are given a fixed meaning in your formalism (equality predicate or integer type), everything else is just an identifier bound by some quantifier, explicitly or implicitly, up in the scope. And finally, we need to break our formalizations into many small pieces, to keep the proof tasks within reach of automated provers.

This has led us to quite a minimalistic system of modules, which are simple collections of specifications and code, with only two basic operations: (a) link module $A$ from module $B$ so that $B$ can have access to the contents of $A$, and (b) put a fresh copy of module $A$ inside module $B$, while replacing some symbols introduced in $A$ with the symbols from $B$. The second operation we call module cloning, and it turned out to be surprisingly (not that surprisingly, if one comes from classical logic) versatile. One of the first cloning instructions we wrote was in the standard library of integers, where we imported the ring axioms by cloning the generic library of rings, replacing the abstract domain $t$ with int, abstract function plus with + , etc. We did not need to say that the module of generic rings was a functor parametrized by that type and those operations. Instead we simply declared an abstract type and three abstract functions on it. And Why3 allows us to instantiate any abstract symbol (or none at all) when cloning a module, on condition that we respect its properties.

Module cloning can help us create abstraction barriers. Write a module $A$ with abstract types and abstract functions, described only by their specifications - this is your interface. Client code may link to $A$ or clone it (to have a fresh instantiated copy), and be verified without knowing anything about the implementation. Write an implementation - a module $B$ with fully defined types and fully implemented operations, and then clone $A$ into $B$ while instantiating every abstract symbol from the interface with its implementation. Why3 will check 
the types and the side effects, and will generate verification conditions for you to prove in order to ensure that the implementation respects the interface.

Module cloning can help us implement generic code. Rings and integers cited above are just one example. Write a module $A$ with all the parameters as abstract symbols - this is your functor. State (and prove) all the generic properties you may need. Clone $A$ into the client code while instantiating the abstract symbols with concrete types and concrete operations. Why3 will transfer all the properties you have proved in the generic module to the client code without requiring you to reprove them.

Below, we present the modules in WhyML (Section 2) and show their use on an example of a Bloom filter library (Section 3), where proofs performed in minimal contexts lead us to a fully implemented correct-by-construction $\mathrm{C}$ program (Section 4). The complete formalization of this case study is available at the companion web page http://why3.Iri.fr/isola-2020/. Our account, though detailed, stays informal: we bring up the soundness properties of the framework but do not try to prove them.

\section{WhyML Modules}

A building block of a WhyML development is a declaration. A declaration can introduce a data type, a mathematical symbol, a logical proposition or a program function. Some declarations provide full information about the symbols they introduce: the structure of a data type is fully exposed, a mathematical symbol is given a sound definition, a proposition is proved, a program function is implemented. Other declarations give us a partial view: we only get to know some fields of a data type, a mathematical symbol is only given a name and a type signature, a proposition is posited without a proof, and a program function shows its specification but the actual implementation is unknown. Mixing concrete and abstract declarations is best suited for program verification, as we get to freely choose the level of abstraction for each element involved. Of course, if we intend to obtain executable code at the end, we must be able to refine the abstract portions into concrete implementations, while preserving the properties obtained through proof.

Declarations are structured using scopes and modules. Scopes help us to manage namespaces. Let us say, we declare a function symbol $f$ in a scope $\mathrm{S}$ :

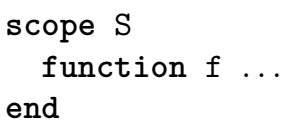

After closing the scope $\mathrm{S}$, we can refer to $\mathrm{f}$ by using a qualifier:

lemma L: ... S.f ...

or by temporarily opening $\mathrm{S}$ inside a WhyML expression:

predicate $\mathrm{p}=\ldots \mathrm{S} .(\ldots \mathrm{f} \ldots) \ldots$ 
As for the module $\mathrm{Q}$ in the same example, it copies the contents of $\mathrm{M}$ while replacing every occurrence of the type $t$ with int. Since type $t$ is abstract, this substitution is allowed. Still, if $M$ contains any axioms about $t$, they may come in contradiction with the properties of type int (e.g., $t$ could be axiomatized as a finite type in $\mathrm{M}$ ), thus creating an inconsistency. This is why all axioms from a cloned module appear by default as lemmas in the cloning module, obliging the programmer either to prove them or to deliberately override the default.

Cloning a module does not affect the symbols that were added to it through the use command. For example, if we clone module N, we get access to the same type $\mathrm{t}$ and function $\mathrm{h}$ as if we have used module $\mathrm{M}$ directly. Informally, one can see use as creating a window into another module. On the other hand, the symbols introduced with clone belong to the cloning module (and Why3 does actually put the instantiated declarations inside the cloning module), and thus can be further instantiated during subsequent clone commands. For example, one can write clone $\mathrm{P}$ with type $\mathrm{M} . \mathrm{t}=$ real.

When we use or clone a module, we introduce new symbols to the logical context and thus, new names. In their shortest form, with no modifiers, both use and clone will put these names in a new scope, named after the module in question, and import that scope. Operations use export and clone export do not open a new scope, and put all the new names in the current namespace instead. For example, module P above can be equivalently written as follows:

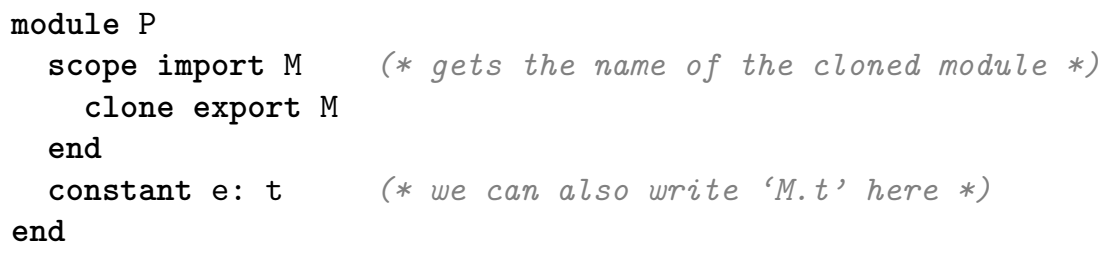

We can choose a different name for the new scope by writing use $\mathrm{M}$ as $\mathrm{A}$ or clone $\mathrm{M}$ as $\mathrm{B}$. In this form, the new scope is not imported automatically:

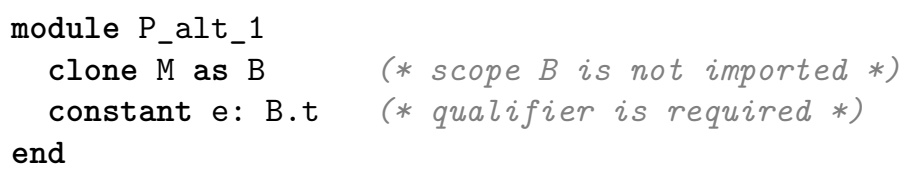

unless we add the import modifier:

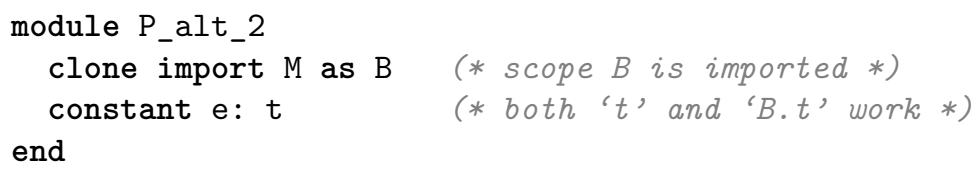

It is important to note that module names can only appear in use or clone operations. In particular, it is impossible to refer to a symbol from a module that has not been added to the current context either through use or through clone. Once it is done, the scope structure will determine the fully qualified name for each symbol that came with that module. 
In what follows, we discuss in more detail various aspects of cloning, paying most attention to the checks and verifications required to ensure the soundness of symbol instantiations. The cloning mechanism guarantees that all properties that have been established in the module being cloned — proved lemmas, verified program contracts, etc. - stay valid after instantiation and can be incorporated into the cloning module without creating a contradiction. This does not mean that cloning a module is always a conservative extension: as we have seen earlier, Why3 does not guarantee that the instantiated axioms of the cloned module are consistent with the current logical context (which is why it incites the programmer to prove them after instantiation). However, whatever has been proved in the cloned module must stay provable after cloning.

We call "original" the module being cloned and the symbols declared in it: type symbols, mathematical symbols, program symbols, etc. The substitution in a clone operation we call a "refinement", the original symbols on the left-hand side being "refined", and the ones on the right-hand side, which replace the originals in the cloned declarations, being "refining". Symbols that are given a full definition in the original module cannot be refined, and are simply transferred into the new context. Their definitions, however, are still instantiated with respect to the cloning substitution, similarly to how in module $\mathrm{Q}$ above, type $\mathrm{t}$ is replaced with int in the signature of the cloned function $\mathrm{h}$.

Type declarations. Fully defined types in Why3 are sum types, non-private records, type aliases, and special numeric types:

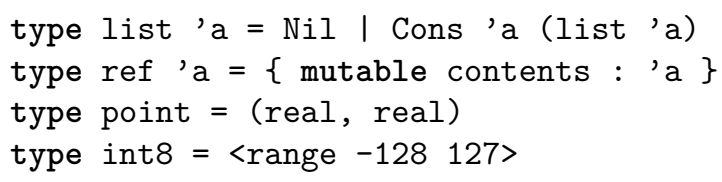

Being fully defined, these types cannot be replaced by cloning instructions. The only refinable types are private records:

type queue 'a $=$ private $\{$ ghost mutable elts: list 'a $\}$

WhyML programs can read the values of private records' fields, but cannot directly construct such records or modify their mutable fields through a direct assignment. Instead, a module that declares a private type like queue should also declare functions to create and manipulate the objects of this type: allocate a new empty queue, add an element to a queue, etc. These functions ought to be implemented in the refining modules that provide a full definition for the type.

A type without definition is considered to be a private record with no fields:

type $\mathrm{t}$ (* the same as 'type $t=$ private \{\} ' *)

A private type whose fields are all ghost (meaning that they can only be used in specifications and in ghost computations, but cannot influence the observable program behaviour) is called "abstract". For example, the definition of type queue above can be equivalently written as follows:

type queue 'a = abstract $\{$ mutable elts: list 'a $\}$ 
Both private and non-private records can be equipped with a type invariant:

type clock = abstract $\{$ mutable $\mathrm{h}$ : int; mutable $\mathrm{m}$ : int $\}$

invariant $\{0<\mathrm{h}<24 \wedge 0<\mathrm{m}<60\}$

A type invariant is essentially an axiom that restricts possible values of the fields of a record type. Only the variables representing these fields are allowed to be free in the invariant; the quantifiers over the new type are also forbidden. Why3 requires type invariants to be satisfiable and generates appropriate proof obligations. Private records, records with mutable fields, and records with type invariants cannot be recursive in WhyML.

A cloning operation can instantiate a private record with a different type. The following restrictions apply:

1. The refining type must have the same number of type parameters as the original type.

2. All fields of the original type must be present in the refining type and have the same type. Here, as before, "the same type" is meant modulo instantiation: that is, if the field's type in the original record is ref $t$ and the cloning substitution replaces $t$ with int, the corresponding field in the refining type must have type ref int.

3. A mutable (respectively, immutable) field in the original type must be mutable (respectively, immutable) in the refining type.

4. A ghost field in the original type may become non-ghost in the refining type but not vice-versa.

5. New fields can be added, which can be mutable and/or ghost. Mutable fields, however, can only be added when the original type is explicitly declared as mutable or has mutable fields of its own.

6. The (instantiated) original invariant must hold for each value of the refining type; Why3 generates an appropriate proof obligation. One possible way to satisfy this requirement is to include the original invariant in the invariant of the refining type.

7. An original field with a mutable type that is not mentioned in the original type invariant cannot occur in the invariant of the refining type either.

The last item deserves some discussion. Let us consider the following declaration:

type ptr 'a $=$ private $\{$ segment: array 'a;

mutable offset: int $\}$

and a variable $\mathrm{p}$ of type ptr. Since ptr is private, modification of the mutable field p.offset is only possible through abstract functions operating on values of type ptr. What about p.segment, an immutable field containing a mutable value? One possibility is to treat it in the same way as p.offset, that is, to forbid direct modification of the array. Another is to allow writes into p.segment. In the latter case, however, we must ensure that any such write does not break the invariant of the ptr type. The problem, of course, is that ptr is a private type and its invariant can be strengthened during refinement. Since we do not know 
the full invariant of ptr right now, we cannot formulate an invariant preservation condition for the writes into p.segment. We can work around this problem by forbidding to constrain the values of the segment field in the current and all future type invariants of ptr, so that no state of p.segment can break the integrity of $\mathrm{p}$. An easy way to ensure this is to forbid mentioning the field in the invariant altogether.

Thus, the presence or the absence of a field with a mutable type (such as segment) in the type invariant of a private type serves as an indication of the user intention: If the field is mentioned in the type invariant (even in a trivially tautological way, like segment $=$ segment), then it becomes non-modifiable ${ }^{2}$; otherwise, it can be written into, but must not appear in the invariants of the refining types, ensuring that modifications are always safe.

Mathematical functions and predicates. Here, the rules are simple, because functions and predicates in WhyML are either provided with a (consistent, total, and unambiguous) definition:

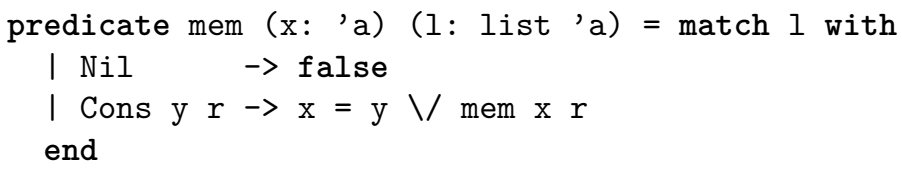

or declared as abstract symbols, with only their name and type signature:

function length (s: string) : int

The defined functions and predicates cannot be refined and their definitions are simply transferred to the current module. An abstract function or predicate is refinable, and the refining symbol must have the exact same type signature modulo instantiation.

For example, the following module clones module $\mathrm{M}$ above, and refines both type $t$ and function $h$ :

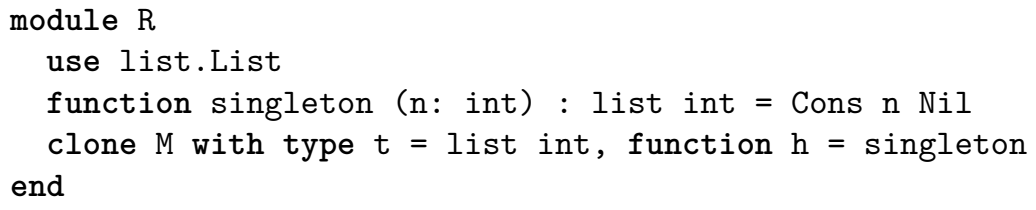

Refinement of functions and predicates does not produce proof obligations.

Logical propositions. Axioms, lemmas, and goals are not refinable: they cannot be replaced with some other propositions. However, Why3 allows the programmer to specify how they should be treated in the cloning module.

The goals in the original module are not transferred to the current module at all. Indeed, they have already been proved in the original module and thus

\footnotetext{
${ }^{2}$ In this case, due to the specifics of state handling in WhyML, not even abstract functions are allowed to announce a potential write in the segment field, which limits the usefulness of this kind of construction. This may be relaxed in the future.
} 
do not need to be reproved after instantiation. And since they are not added to the logical context as premises (contrary to lemmas), the cloning module has no need for the original goals.

The original lemmas are cloned as lemmas; however, since they have already been proved in the original module, Why3 will not generate a proof obligation for them. If we do not want to keep a cloned lemma as a premise in the logical context (e.g., because it duplicates an existing premise), we can "recast" it as a goal by writing with goal $\mathrm{L}$ in the cloning substitution (where $\mathrm{L}$ is the name of the original lemma). Then lemma $\mathrm{L}$ will not be copied to the current module.

Axioms require caution, because, as we have noted above, simply copying an original axiom into the new context may create an inconsistency. To prevent this from happening, WhyML clones axioms as lemmas by default (and generates proof obligations for them), and the programmer must explicitly specify which axioms of the original module are to be kept as axioms:

clone relations.PreOrder with axiom Refl, axiom Trans

This instruction clones the PreOrder module from the file relations.mlw from the standard library of Why3. It adds to the current module declarations of a new abstract type $t$ and a new binary relation $r e l$ on $t$ together with the axioms of reflexivity and transitivity of rel.

When cloning modules with numerous axioms, listing all of them would be tedious. Therefore, WhyML provides a shortcut with axiom . which preserves every axiom in the original module unless it is converted into a lemma or a goal elsewhere in the cloning substitution.

Program functions. Only the abstract program functions, characterized by their type signature and their contract, can be refined in a cloning substitution. However, due to the large variety of possible side effects in the original and refining functions, the required checks are rather complex. For example, consider the code in Figure 1 (we omit the references to the standard library of lists). The Queue module declares an abstract type of mutable queues and an abstract enqueuing function. The TwoListQueue module implements the queue type and the enqueue operation, and then clones Queue, refining the two symbols.

After checking the correctness of the type refinement (remember that in an abstract record all fields are ghost and thus field elts is allowed to stay ghost in the implementation), Why3 proceeds to the refinement of enqueue.

The procedure starts with instantiating the prototype of the original abstract function. This step does not take the refining function into consideration; in fact, the same rules are applied when we simply transfer an abstract function into the cloning module without refining it. Prototype instantiation is non-trivial because the types in the original type signature, notably those involved in the side effects, may have been refined, revealing new mutable fields and new fields with mutable components. This is the case in our example: the modified parameter $q$ has gained two new mutable fields, front and back.

Why3 applies the following rules when instantiating the "writes" annotations for the modified mutable values whose type is refined (remember that all side 


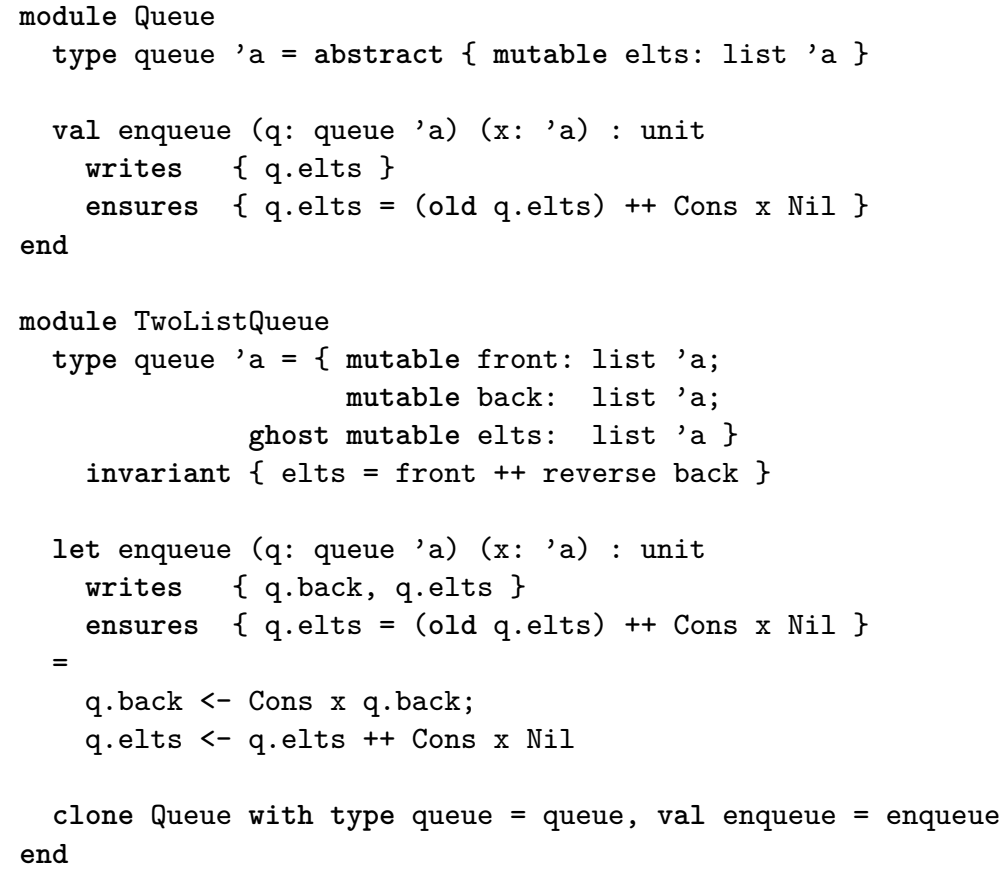

Fig. 1. Queues: interface and implementation.

effects in these annotations are latent and do not have to actually happen in any implementation):

1. All original mutable fields marked as written in the original prototype are considered written in the instantiated prototype.

2. All original fields not marked as written in the original prototype are not considered written in the instantiated prototype.

3. All new mutable fields are considered written in the instantiated prototype.

4. All mutable components of the new fields are considered written in the instantiated prototype.

Mutable values that are not modified in the original prototype, are not modified in the instantiated prototype either, regardless of how their type is refined.

According to these rules, the instantiated prototype of the original function enqueue is as follows:

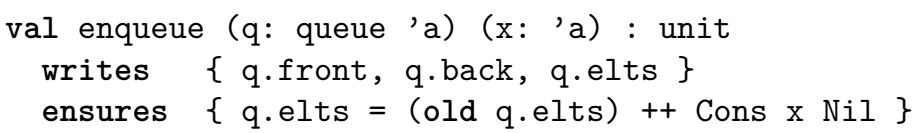

Indeed, q.elts is considered written, as it was already marked as such in the original prototype (rule 1). The fields front and back are added to the write 
effect, since they are new mutable fields in a modified parameter q (rule 3). If the added fields front and back were not mutable but had a mutable type (say, array 'a), they would also appear in the instantiated effect by rule 4 .

To sum up, the instantiated effect annotation stays the same with respect to what is known by the original module. However, each announced write effect extends to all added fields of the affected values. This allows the implementations of the original enqueue function to modify the new fields front and back.

Now that we have the instantiated prototype of the original abstract function, we need to compare it with the proposed refinement and verify that the instantiation is legal. This requires multiple checks:

1. The type signatures must coincide.

2. Ghost parameters of the original should be ghost in the refinement (an implementation cannot depend on ghost data passed from the client code).

3. Ghost results of the refinement should be ghost in the original (an implementation cannot pass ghost data to client code unbeknown to it).

4. The refining function must not have effects unlisted in the instantiated prototype of the original.

5. The refining function must not create memory aliases that are not required by the original.

6. The refinement must satisfy the instantiated contract of the original, that is, have a weaker (or equivalent) precondition and a stronger (or equivalent) postcondition.

In order to check these conditions, Why3 creates and verifies (and then throws out) a WhyML function whose specification comes from the instantiated original prototype and whose implementation consists in calling the refining function with the same parameters. The type-checking system of Why3 and its verification condition generator perform the necessary checks and produce an appropriate proof obligation for the last item. In the case of enqueue, this proof obligation is an easily provable tautology.

While the rules for prototype instantiation introduce new latent write effects, these effects only concern the values that are already marked as modified in the original prototype, and they are limited to the new fields. Since a caller of the abstract function only knows the fields in the original type declaration, it can only observe a modification in the new fields as a non-specific change of the whole value - which is covered by the effect annotation in the original prototype.

It is crucial for the soundness of cloning that no aliases exist between the values accessible to the caller and the "hidden state" represented by the added fields. Such an alias can only be created through a refinement of an abstract program function in the original module, and this is prevented by rule 5 above.

In the next section, we show how to use modules in a fully developed example, going from abstract specifications to executable $\mathrm{C}$ code. In particular, we demonstrate how module cloning expresses: (a) the relation between an interface and an implementation; (b) the relation between a generic module and its parameters; and (c) specialization of a generic module. 


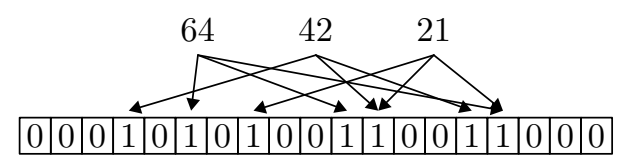

Fig. 2. A Bloom filter for integers, using $m=19$ and $k=3$.

\section{Example: Bloom Filters}

A Bloom filter [4] is a data structure that implements a set and provides two operations: one to insert an element into the set and one to query the presence of an element in the set. The latter must always give a correct positive answer for elements that have been indeed inserted into the set, but it may return a false answer for the elements not in the set. In other words, false positives are allowed but false negatives are not.

A Bloom filter makes use of a bitmap (a Boolean array) of a given size $m$ and of $k$ hash functions $h_{1}, \ldots, h_{k}$ mapping the elements to integers between 0 and $m-1$. When inserting an element $x$, we set the bits at indices $h_{1}(x), \ldots, h_{k}(x)$. When querying the presence of $x$, we return true if and only if all bits at indices $h_{1}(x), \ldots, h_{k}(x)$ are set.

Figure 2 illustrates a Bloom filter for integer elements where we use an array of 19 bits and 3 hash functions $h_{1}(x)=34 x, h_{2}(x)=55 x$, and $h_{3}(x)=89 x$ (all considered modulo 19). We insert three elements into the set, namely 21, 42 , and 64 . It results in seven bits being set (bits at indices $3,5,7,10,11,14$, and 15 in the array). If we now query the filter for the element 82 , it reports that it is not in the set. Indeed, element 82 is mapped to bits 2, 7, and 14 and, though bits 7 and 14 are set, bit 2 is not and thus 82 does not belong to the set. But if we now query the filter for the element 80 , it checks for bits 3,11 , and 14 , which are all set, and thus reports that 80 belongs to the set. This is a false positive. If we query the filter for all elements between 0 and 99, it reports 17 positives: the three elements we added and 14 false positives. For the remaining 83 elements, we know for sure they do not belong to the set.

Despite being imprecise, a Bloom filter is a genuinely useful data structure. One good application is the following. Say we are implementing a storage whose operations are expensive, because they involve disk or network access. A Bloom filter can be conveniently placed between the storage and its client. When an element is added to the storage, it is added to the filter as well. Whenever the storage needs to be queried, we first query the filter. If the filter reports that the element is not in the storage, the answer is guaranteed to be correct and we avoid a costly operation. By themselves, Bloom filters are efficient data structures, in both space and time. With suitable choices of $m$ and $k$, a Bloom filter can achieve an error ratio less than $1 \%$ with less than 10 bits per element [13].

It is worth pointing out that unlike a traditional hash table, a Bloom filter cannot be resized to accommodate an increasing number of elements. Indeed, the elements themselves are not kept in the Bloom filter and thus there is no way to rehash them into a larger array. This means that we must make an 


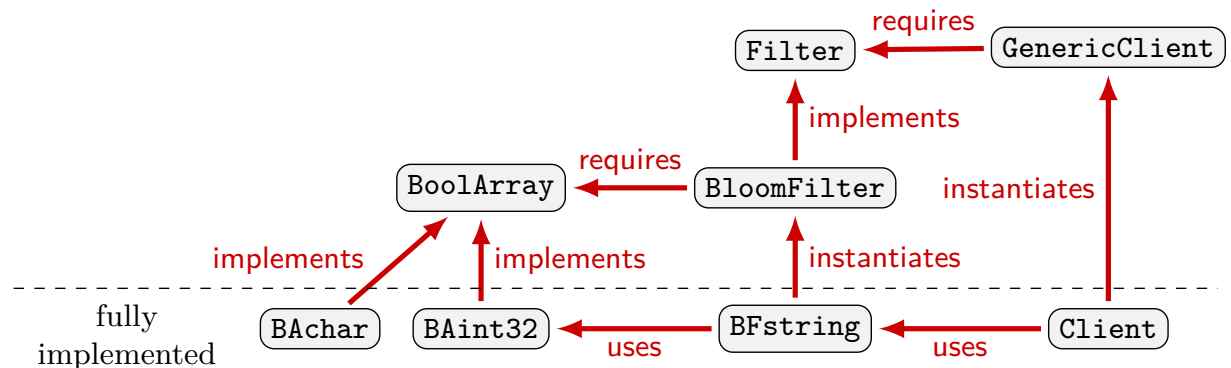

Fig. 3. Bloom filters in Why3: the module map.

estimation of the expected size of the element set in advance, and pick the value of $m$ accordingly. Similarly, there is no way to remove an element from a Bloom filter. Indeed, by clearing the bits corresponding to an element, we could remove other elements from the set, which would make the filter unsound. This is not a problem, since removing an element from the actual storage without updating the Bloom filter would merely lead to another false positive, which is allowed. If need be, the filter can be reconstructed from the storage on regular intervals, without compromising the asymptotic complexity.

Let us implement a Bloom filter with Why3. Our final objective is to get a verified $\mathrm{C}$ library of Bloom filters. We decompose the code into eight modules, shown in Fig. 3. Notice that the verbs "requires", "implements", and "instantiates" are all realized using the clone operation, as will be demonstrated throughout this section. Here is a short overview of the diagram:

- On the left side of the figure, we have three modules related to Boolean arrays. Module BoolArray is an interface and modules BAchar and BAint32 are two implementations of this interface. The former implements a Boolean array rather naïvely, using one byte per element. The latter uses an array of 32-bit integers, using one bit per element.

- On the central part of the figure, we have three modules related to filters. Module Filter is an interface. It provides an abstract data type and three operations create, add, and mem. Module Bloomfilter implements Bloom filters on the basis of various parameters: an implementation of Boolean arrays, values for $m$ and $k$, a data type for the elements, and a set of hash functions. Then module BFstring instantiates all these parameters to get a fully implemented Bloom filter for strings.

- Finally, on the right side of the figure, we have two modules to make a quick test of the library. Module GenericClient uses the interface Filter to build a filter and perform a few additions and membership queries. Then module Client instantiates this generic client using module BFstring.

The four modules at the bottom of the figure are fully implemented and can be translated to compilable $\mathrm{C}$ code (this is described in the next section). 
Boolean arrays. We start with an interface BoolArray, which declares a type $t$ together with three operations create, get, and set.

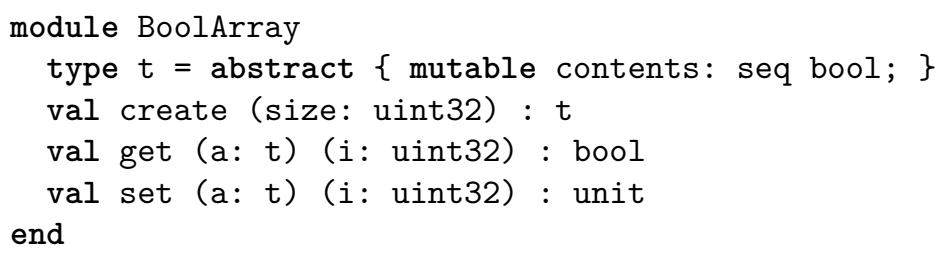

We omit various details here, such as the modules imported from the standard library and the contracts for the three operations. The type $t$ is an abstract record data type, with a single field named contents. Since all fields of an abstract type are ghost, the field contents can be used within any specification element, such as a function contract, but cannot be used in actual computation in the code. In other words, it serves as a model for the type $t$, but not as a part of its implementation. This model is a sequence of Boolean values (type seq, from Why3 standard library, can be seen as a purely applicative array) and this is all we need to provide suitable contracts to our three operations. For instance, operation get is given the following contract:

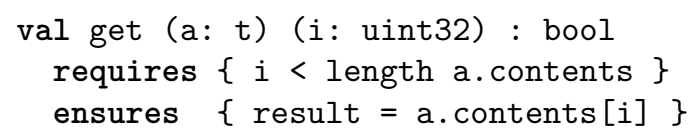

For convenience, WhyML allows us to declare contents a coercion symbol, so that we can write simply a instead of a.contents. Module BoolArray does not incur any verification condition.

We now provide two different implementations of this interface. We start with a rather simple implementation with one byte per bit. We do this in a separate module BAchar. It also contains declarations for a type $t$ and three operations create, get, and set.

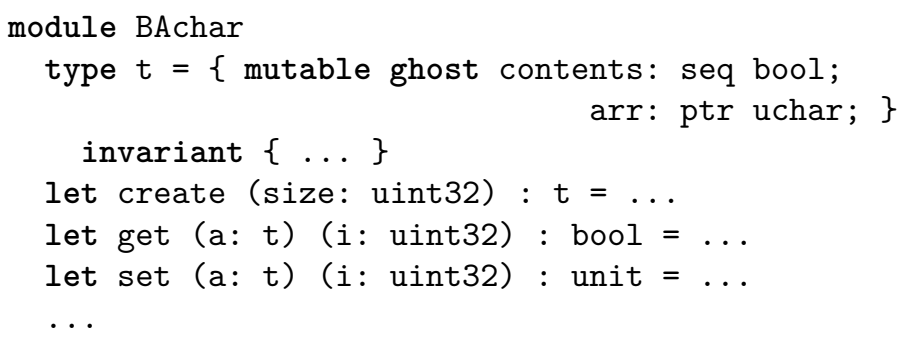

This time, however, our types and functions are fully implemented. Type $t$ is still a record data type with a ghost field contents. But it also contains a non-ghost field arr that holds a pointer to an array of bytes (type uchar from Why3 standard library). A gluing invariant (omitted here) makes the connection between field contents (the model) and field arr (the implementation). The type $t$ is not abstract anymore, which means we are now allowed to construct instances of that type. This is precisely what function create does. 
Operations create, get, and set are now given definitions (omitted here). Their contracts are identical to those of module BoolArray. In particular, they only refer to field contents. Their definitions, of course, do make use of field arr. Why3 generates suitable verification conditions for these three definitions to be correct with respect to their contracts.

Finally, we show that module BAchar is indeed an implementation of the interface BoolArray. This is done with the help of a clone instruction:

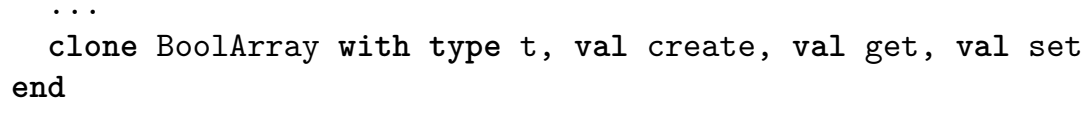

Here, we use a syntactical shortcut that allows us to write only the left-hand side of the substitution when the refining symbol has the same name as the original. That is, we substitute the type $t$ of module BoolArray with the type $t$ we just defined, and similarly for the three operations.

This clone command generates several verification conditions. They are all rather trivial, as there is no invariant on type BoolArray.t and the contracts for the three operations are the same in the interface and the implementation.

Apart from this last clone, module BAchar is completely independent of BoolArray. The clone instruction matches the definitions in BAchar to the declarations in BoolArray and verifies that the former can indeed serve as an implementation of the latter. It is perfectly possible for a module to implement several different interfaces.

We also provide a second implementation of BoolArray in a module called BAint32. It is a more efficient implementation that uses an array of 32-bit integers, where each element packs 32 Boolean values.

Filters. We proceed in a similar way for filters, though using two layers of refinement instead of one. We start with an interface, Filter, which declares types for elements and filters and three operations:

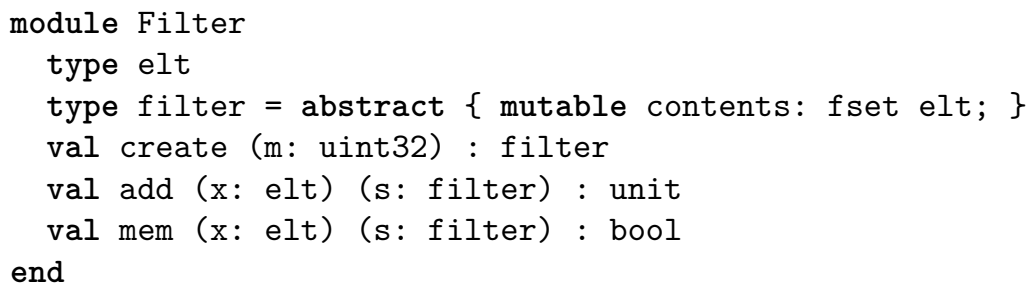

This is similar to what we did earlier with module BoolArray. Here, the contents of type filter is modeled using a finite set. Then we implement Bloom filters in a second module BloomFilter. We start by introducing parameters for the type of elements and the family of $k$ hash functions.

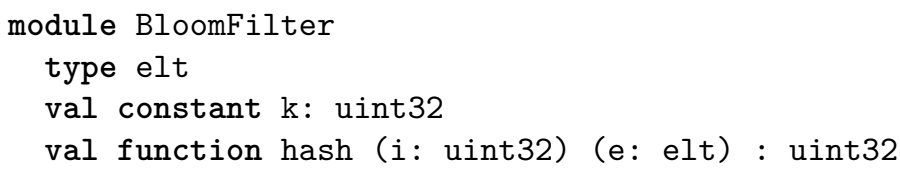


The individual hash functions are identified with an index $\mathrm{i}$ in $0 . . \mathrm{k}-1$. Then we move to the implementation of type filter. For that, we need a Boolean array, and so we bring a copy of BoolArray into the context.

clone BoolArray

It is worth pointing out that this module is merely an interface for Boolean arrays. This means that our implementation of Bloom filters does not depend on a particular implementation of that data structure, and can be instantiated to use any of them. We can now define type filter on top of BoolArray.t.

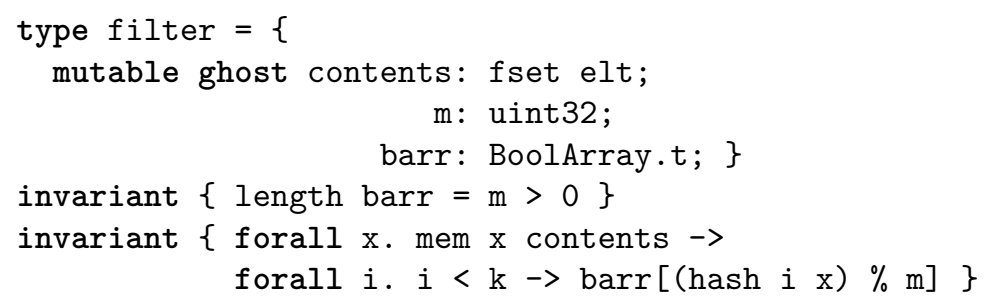

The gluing invariant makes the connection between the model field contents and the implementation fields $\mathrm{m}$ and barr. Now we can implement the three operations over Bloom filters:

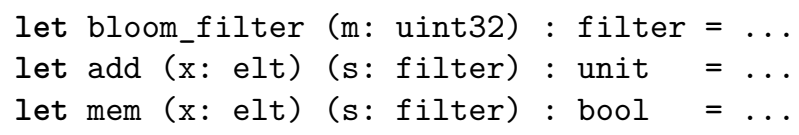

Note that, despite being defined, these functions still depend on parameters elt, $\mathrm{k}$, and hash. Thus, they are not executable.

Last, as we did with module BAint32, we check that this module refines module Filter, using a clone command.

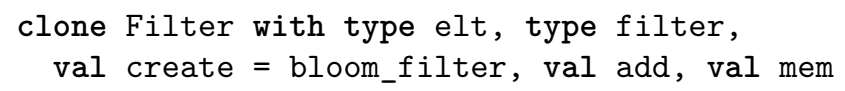

Again, this generates VCs that are all easily discharged.

In order to obtain executable code, we further refine module BloomFilter to produce a filter for strings. Here, we choose to use three hash functions.

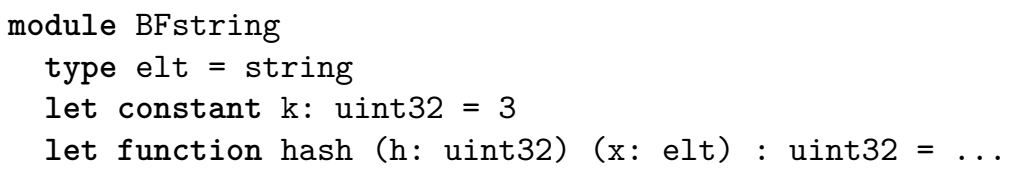

The actual implementation of hash, omitted here, is based on Fowler-Noll-Vo hash functions, following Louridas [12]. The remaining part is a clone command to instantiate BloomFilter with these parameters and with module BAint32:

use BAint32

clone export Bloomfilter with val $k$, type elt, val hash, 


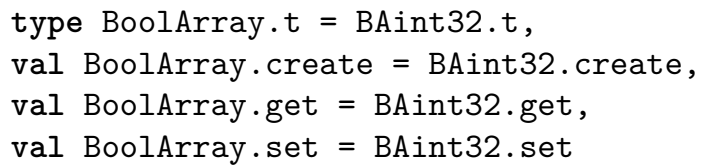

Though for Why3 this clone command is no different from the previous two, from the programmer's point of view it is of a rather different flavor. Instead of claiming that module BFstring implements BloomFilter, it rather instantiates module BloomFilter with actual parameters. Notice that we write export in order to have the Bloom filter operations in the top namespace of BFstring.

Client. We conclude this example with a tiny client code. The main purpose is to check the usability of our contracts before going any further. We start with a client for module Filter, which we instantiate on string elements.

module GenericClient

clone Filter with type elt = string

Then a test function builds a filter of a given size, inserts some strings, and checks for membership:

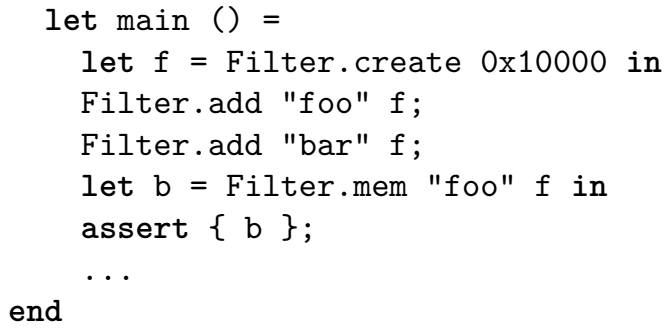

Once this is done, and verified, we can clone this generic client with a specific implementation of Filter, namely module BFstring we built earlier.

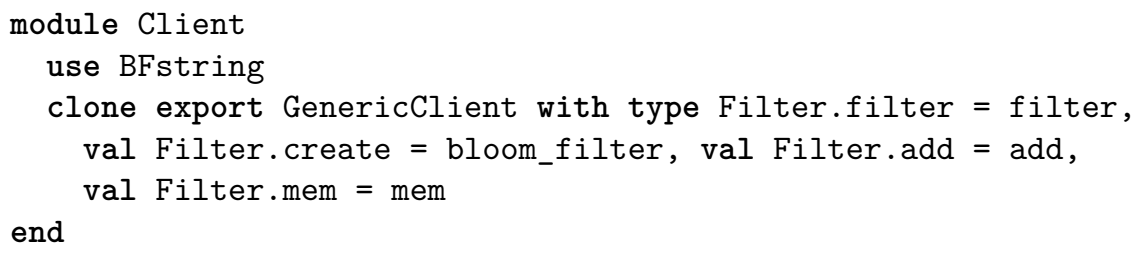

This verification passes, too, as we have already checked that BFstring implements Filter. (As for now, Why3 unnecessarily generates the same VCs a second time; this will be improved in the future.) Module Client is fully implemented and we will be able to translate it into executable $\mathrm{C}$ code, as shown in the next section. If we look again at the right-hand size of Fig. 3, we can see that the correctness of the Client module is ensured by the correctness of GenericClient and the fact that BFstring correctly refines Filter. A similar relation exists between modules BFstring, BloomFilter, BAint32, and BoolArray. 


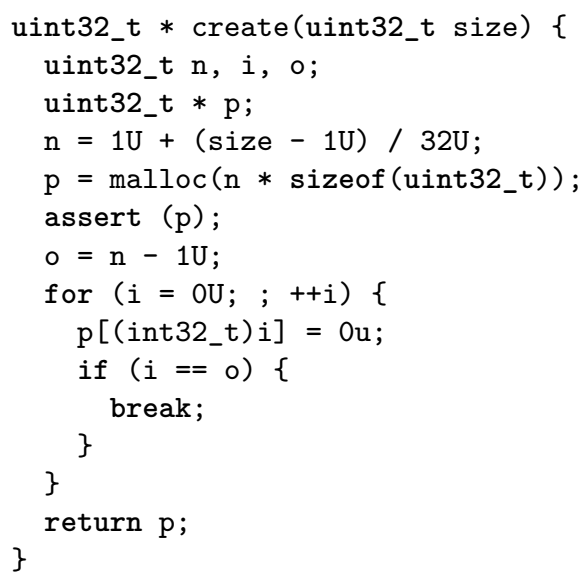

Fig. 4. Generated C code for function create from module BAint32.

\section{C Library}

Once verification is complete, Why3 can automatically translate WhyML code to $\mathrm{C}$ [14]. The resulting $\mathrm{C}$ code is composed of three files:

- baint32.c, a translation of module BAint32;

- bfstring.c, a translation of module BFstring;

- client.c, a translation of module Client.

File bfstring.c makes use of functions from baint32.c and file client.c makes use of functions from bfstring. c. Each $\mathrm{C}$ file comes with a corresponding header file (.h). These files are available at http://why3.Iri.fr/isola-2020/. Figure 4 contains the $\mathrm{C}$ code for function create from file baint 32 .c, resulting from the translation of function create from module BAint32. We can make two comments regarding this code. First, assert is used so that we can assume that the value returned by malloc is not NULL in the following, without having to test it. This is reflected on the Why3 side with an assert function that ensures (in its postcondition) that $\mathrm{p}$ is not NULL. Second, the rather unusual form of the for loop, using a break statement, ensures that even a loop up to the maximum representable value is sound with respect to the WhyML semantics. In this case, the loop is bounded by $n-1$ so a traditional loop would be fine but Why3 does not make any effort to figure that out.

It is worth pointing out that each generated header file exposes all declarations from the corresponding WhyML module. For instance, file baint $32 . \mathrm{h}$ declares the functions create, get, and set, as expected, but also "internal" functions one_bit, bit_set, and set_bit. Similarly, file bfstring.h declares the structure filter and the functions bloom_filter, add, and mem, but also the global variable $\mathrm{k}$ and the functions hash and bit. We translate all declarations 
because the translation is made on per-module basis. In WhyML, modules do not have dedicated interfaces and any module using module Baint32 has access to all of its declarations. Thus, this ability must not be lost in translation.

An argument can be made that it is not crucial to ensure any abstraction barrier in the translated code since we have already made use of it on the WhyML side. This argument is less applicable when we develop a verified library for the target language, which is the case of bfstring. Indeed, the development will be pursued in the target language and thus it would be nice to hide the translated code behind a suitable interface. The simplest way to achieve it is just to remove unnecessary declarations from the generated header files. Finally, when translating to $\mathrm{C}$, the whole discussion is moot since there is no proper encapsulation in $\mathrm{C}$ (it is always possible to bypass header files).

\section{Related Work}

The idea of conducting verification through stepwise refinements is not new. It is at the basis of Abrial's B method [1] for instance. In this context, abstract machines, which can be seen as interfaces, are gradually refined into fully executable machines, which are implementations. This is quite close to what we do: for instance, when we start with an interface Filter and refine it into an implementation BFstring in two steps. Proper modularity is also offered by the $\mathrm{B}$ method, as a machine is referring to the abstract version of another machine (its interface) and not to its refinements. Again, this is similar to what we do, for instance with our GenericClient referring to the interface Filter. Yet, there are fundamental differences between B machines and Why3 modules, the main being that B machines are state machines. Though Why3 modules can definitely be used to specify and implement state machines, they are not limited to this usage. Why3 modules may provide data types (as Boolean arrays and filters in our example) and this has no counterpart in the B method.

Abstraction and genericity are handled in programming languages in various ways. Most of these solutions can be readily used or adapted for use in program verifiers. When a program verifier is built for an existing programming language, such as Java for instance, it is natural to apply the abstraction and genericity mechanisms (e.g., object-oriented programming, visibility modifiers, generic types) to the specification/verification level. This is done in tools such as VeriFast [7] or KeY [2] for instance. When a program verifier is providing its own programming language, it is nonetheless possible to reuse mechanisms from the programming community. The Coq proof assistant, for instance, implements both a module system inspired by that of OCaml $[11,5]$ and type classes inspired by those of Haskell $[17,16]$.

Why3 modules are not a direct implementation of a concept from any programming language. Yet, they have obvious connections with traits [15] and mixins [3], even if they are not cast in some object-oriented context. Indeed, Why3 modules mix declarations and definitions, may require parameters to come with some operations (by cloning suitable "interfaces"), and may provide new def- 
initions on top of these parameters (in modules to be later cloned in suitable contexts). The comparison stops at some point, however, as Why3 modules are not centered around types. A parameter of a Why3 module can be a constant, a function, etc., which means more flexibility. On the other hand, Why3 modules cannot be used to require that a type parameter of a polymorphic type or function provides some operations, contrary to traits or type classes.

Closest to our work is likely to be Dafny [9], where modules are used to organize the namespace and to restrict visibility of symbols or symbol definitions [10]. Thus it provides adequate abstraction during the verification of client code, though this is done by hiding implementation details rather than having the client exposed to an interface only. There is a notion of module refinement in Dafny [8]. As in Why3, it allows declarations to be refined with definitions and it permits data refinement, though it is class-based in Dafny and record-based in Why3. Dafny goes a step forward in program refinement, allowing reduction of nondeterminism in program statements during refinement.

\section{Conclusion}

We have shown how abstraction and genericity are provided in the Why3 program verifier through a notion of modules and a module cloning operation. The latter performs a partial substitution on a module, replacing some of its abstract declarations with concrete ones, and generates suitable verification conditions to guarantee correctness. In this paper, we demonstrated this mechanism on a library of Bloom filters, using several modules and refinement steps.

The module system of Why3, despite being usable (and extensively used), can still be improved in several regards.

First, we should avoid redundant verification conditions (such as ones generated for the clone instruction in the Client module) by taking into account the previously made refinements. In practice, these redundant VCs are usually easy to discharge, but it is preferable not to produce them at all.

Second, we should add support for scope-level cloning substitutions, which would allow us to write simply clone GenericClient with scope Filter = BFstring, and avoid long and tedious enumeration of individual refinements.

Third, it would be convenient to annotate an "implementation" module with its designated interface, e.g., by writing module BAint32 : BoolArray. This notation should automatically add an appropriate cloning instruction at the end of BAint32, ensuring that it indeed refines BoolArray. Furthermore, any subsequent use of BAint32 in the client code should only add to the logical context the contents of BoolArray, acting as an abstraction barrier (of course, translation into executable code would still use the concrete definitions from BAint32). This can be achieved by implicitly replacing such use instructions with cloning of BoolArray, like we did in the Bloomfilter module above; also, renaming substitutions should be applied to ensure symbol sharing where necessary.

Acknowledgments. We are grateful to Claude Marché, Jacques-Henri Jourdan, and Rustan Leino for their insightful remarks and suggestions. 


\section{References}

1. Jean-Raymond Abrial. The B-Book, assigning programs to meaning. Cambridge University Press, 1996.

2. Wolfgang Ahrendt, Bernhard Beckert, Richard Bubel, Reiner Hähnle, Peter H. Schmitt, and Mattias Ulbrich, editors. Deductive Software Verification - The KeY Book - From Theory to Practice, volume 10001 of Lecture Notes in Computer Science. Springer, 2016.

3. Davide Ancona and Elena Zucca. An algebraic approach to mixins and modularity. In M. Hanus and M. Rodrìguez Artalejo, editors, 5th Intl. Conf. on Algebraic and Logic Programming, number 1139 in Lecture Notes in Computer Science, pages 179-193, Berlin, 1996. Springer.

4. Burton H. Bloom. Space/time trade-offs in hash coding with allowable errors. Commun. ACM, 13(7):422-426, July 1970.

5. Jacek Chrzaszcz. Modules in Type Theory with Generative Definitions. PhD thesis, Warsaw University, Poland and Université de Paris-Sud, January 2004.

6. Jean-Christophe Filliâtre and Andrei Paskevich. Why3 - where programs meet provers. In Matthias Felleisen and Philippa Gardner, editors, Proceedings of the 22nd European Symposium on Programming, volume 7792 of Lecture Notes in Computer Science, pages 125-128. Springer, March 2013.

7. Bart Jacobs, Jan Smans, Pieter Philippaerts, Frédéric Vogels, Willem Penninckx, and Frank Piessens. VeriFast: A powerful, sound, predictable, fast verifier for C and Java. In Mihaela Gheorghiu Bobaru, Klaus Havelund, Gerard J. Holzmann, and Rajeev Joshi, editors, NASA Formal Methods, volume 6617 of Lecture Notes in Computer Science, pages 41-55. Springer, 2011.

8. Jason Koenig and K. Rustan M. Leino. Programming language features for refinement. In John Derrick, Eerke A. Boiten, and Steve Reeves, editors, Proceedings 17th International Workshop on Refinement, Refine@FM 2015, Oslo, Norway, 22nd June 2015., volume 209 of Electronic Proceedings in Theoretical Computer Science, pages 87-106, 2015.

9. K. Rustan M. Leino. Dafny: An automatic program verifier for functional correctness. In $L P A R-16$, volume 6355 of Lecture Notes in Computer Science, pages 348-370. Springer, 2010.

10. K. Rustan M. Leino and Daniel Matichuk. Modular Verification Scopes via Export Sets and Translucent Exports, pages 185-202. Springer International Publishing, Cham, 2018.

11. Xavier Leroy. A modular module system. Journal of Functional Programming, 10(3):269-303, 2000.

12. Panos Louridas. Real-World Algorithms: A Beginner's Guide. The MIT Press, 2017.

13. Michael Mitzenmacher and Eli Upfal. Probability and Computing: Randomized Algorithms and Probabilistic Analysis. Cambridge University Press, USA, 2005.

14. Raphaël Rieu-Helft, Claude Marché, and Guillaume Melquiond. How to get an efficient yet verified arbitrary-precision integer library. In 9th Working Conference on Verified Software: Theories, Tools, and Experiments, volume 10712 of Lecture Notes in Computer Science, pages 84-101, Heidelberg, Germany, July 2017.

15. Nathanael Schärli, Stéphane Ducasse, Oscar Nierstrasz, and Andrew P. Black. Traits: Composable units of behaviour. In Luca Cardelli, editor, ECOOP 2003 Object-Oriented Programming, pages 248-274, Berlin, Heidelberg, 2003. Springer Berlin Heidelberg. 
16. Matthieu Sozeau and Nicolas Oury. First-Class Type Classes. In Otmame AïtMohamed, César Muñoz, and Sofiène Tahar, editors, 21th International Conference on Theorem Proving in Higher Order Logics, volume 5170 of Lecture Notes in Computer Science. Springer, August 2008.

17. P. Wadler and S. Blott. How to make ad-hoc polymorphism less ad hoc. In Proceedings of the 16th ACM SIGPLAN-SIGACT Symposium on Principles of Programming Languages, POPL '89, pages 60-76, New York, NY, USA, 1989. ACM. 